\title{
Simulations éléments-finis de la déformation de textiles aux échelles macro et mésoscopique
}

\author{
Philippe Boissea ${ }^{a}$ Nahiene Hamila, Pierre Badel et Emmanuelle Vidal-Salle \\ Université de Lyon, INSA - Lyon, LaMCoS, UMR 5259, 69621 Villeurbanne Cedex, France
}

Reçu le 28 août 2008, accepté le 9 avril 2009

\begin{abstract}
Résumé - La connaissance du comportement mécanique des tissus utilisés comme renforts de composites est nécessaire pour réaliser des simulations de préformage. Cette étude vise à rappeler la spécificité du comportement mécanique des tissus secs et à déterminer ces propriétés mécaniques par des essais virtuels. Pour ceci, des analyses éléments-finis 3D des cellules tissées unitaires sont réalisées. Ces calculs ne sont pas classiques compte tenu de la constitution des fils faits de milliers de fibres de petits diamètres. Les mouvements relatifs possibles des fibres rendent très faible la plupart des rigidités autres que celles de tension. Les spécificités du calcul pour déterminer ce comportement mécanique seront décrites. Plusieurs aspects spécifiques de l'analyse sont détaillés ; particulièrement l'utilisation d'une loi hypoélastique basée sur une dérivée objective utilisant la rotation de la fibre. Les résultats de ces tests virtuels sont comparés à des résultats expérimentaux en particulier obtenus par tomographie X. Enfin ces comportements matériaux sont utilisés dans des simulations macroscopiques dans des cas d'emboutissages à un ou plusieurs plis. Il est discuté de l'importance des différentes rigidités dans l'apparition et la forme des plis.
\end{abstract}

Mots clés: Tissés / textiles / propriétés mécaniques / éléments-finis (MEF)/ mise en forme / méso-macro

\begin{abstract}
Finite-element simulations of textile deformations at macro and mesoscale. The knowledge of the mechanical behavior of fabrics used as reinforcements of composites is necessary to carry out simulations of preforming. This study aims pointing out the specificity of the mechanical behavior of dry fabrics and at determining these mechanical properties by virtual tests. In that goal, 3D finite-elements analyses of the unit woven cells are carried out. These calculations are not standard taking into account the constitution of the yarns that are made of thousands of fibres of small diameters. The possible relative movements of fibres make very weak the rigidities other than those of tension. Specificities of calculation to determine this mechanical behavior will be described. Several specific aspects of the analysis are detailed; particularly the use of a hypoelastic law based on an objective derivative using the rotation of fibre. The results of these virtual tests are compared with experimental results obtained in particular by tomography $\mathrm{X}$. Finally these material mechanical behaviors are used in macroscopic simulations in cases of one and several ply stamping. It is discussed of the importance of various rigidities in the appearance and the shape of wrinkles.
\end{abstract}

Key words: Fabrics / textiles / mechanical properties / finite-element analysis (FEA) / forming / meso-macro

\section{Introduction}

Le comportement mécanique des tissus est complexe compte tenu des mouvements possibles et des interactions entre les fils (échelle mésoscopique) et fibres (échelle microscopique). En dépit des nombreux travaux dans le

\footnotetext{
a Auteur pour correspondance :

Philippe.Boisse@insa-lyon.fr
}

domaine, il n'y a pas de modèle universellement admis qui décrit tous les aspects du comportement mécanique d'un tissu. Les familles de modèles existant viennent de la nature multi-échelle du textile.

Une première famille de modèles considère le tissu comme un continuum anisotrope $[1,2]$, dont le comportement mécanique doit tenir compte de l'influence de la méso-structure tissée. Si ces modèles peuvent être facilement intégrés dans les éléments-finis classiques, 
l'identification des paramètres matériels homogénéisés est difficile, particulièrement parce que ces paramètres changent quand le tissu est soumis aux grandes déformations dues à une mise en forme.

À l'opposé, certains auteurs ont proposé des modèles discrets pour les tissus basés sur une modélisation de chaque fil ou fibre tissés habituellement décrits par des éléments simplifiés tels que les poutres et les ressorts $[3,4]$. Une difficulté importante se situe dans le nombre très grand des composants à l'échelle mésoscopique ou microscopique.

Dans cet article les éléments-finis utilisés sont de type semi-discrets $[5,6]$. Les composants à l'échelle mésoscopique sont considérés (fils, ou cellules tissées). Mais dans le cas de l'approche semi-discrète, ils font partie d'éléments-finis et leurs déformations sont fixées par les déplacements aux nœuds. L'énergie de déformation correspondante est calculée à partir du comportement de tension biaxiale et de cisaillement plan obtenu à partir des analyses 3D à l'échelle mésoscopique réalisées sur une cellule tissée élémentaire.

La première partie de cet article décrit ces analyses mésoscopiques et leur validation par tomographie X. La seconde partie présente un exemple de simulation de mise en forme et montre l'importance des différentes rigidités dans l'apparition et la forme des plis.

\section{Analyse mécanique des renforts tissés à l'échelle mésoscopique}

Réaliser des simulations mésoscopiques des déformations de renforts textiles par la méthode des élémentsfinis requiert un modèle de comportement du matériau «mèche ». Ce comportement est très particulier en raison de la nature fibreuse du matériau que l'on devra pourtant considérer comme continu. Ainsi, la grande rigidité longitudinale des mèches devant les autres rigidités et le comportement transverse de la mèche sont deux aspects importants pour ce type d'analyse.

\subsection{Modèle de comportement mécanique}

Le modèle de comportement adopté ici est écrit dans le cadre de l'hypo-élasticité [7]. Le comportement mécanique est supposé isotrope transverse dans le plan perpendiculaire à la direction des fibres que l'on note $\underline{\mathbf{f}}_{1}$.

Une loi de comportement hypo-élastique a la forme suivante : $\underline{\underline{\boldsymbol{\sigma}}}^{\nabla}=\underline{\underline{\underline{\mathrm{C}}}}: \underline{\underline{\mathrm{D}}}$ où $\underline{\underline{\boldsymbol{\sigma}}}^{\nabla}$ et $\underline{\underline{\mathrm{D}}}$ sont respectivement les tenseurs de taux de contraintes de Cauchy objectif et de taux de déformation et $\underline{\underline{C}}$ le tenseur de comportement. Cette équation est intégrée sur un incrément de temps $\Delta t=t^{n+1}-t^{n}$ en utilisant la formule de Hughes et Winget [8] fréquemment utilisée dans les codes de calcul par éléments-finis :

$$
\left[\boldsymbol{\sigma}^{n+1}\right]_{\mathrm{e}_{i}^{n+1}}=\left[\boldsymbol{\sigma}^{n}\right]_{\mathrm{e}_{i}^{n}}+\left[\mathbf{C}^{n+1 / 2}\right]_{\mathrm{e}_{i}^{n+1 / 2}}[\Delta \varepsilon]_{\mathrm{e}_{i}^{n+1 / 2}}
$$

où $[\mathbf{S}]_{\mathrm{e}_{i}^{n}}$ est la matrice des composantes d'un tenseur $\mathbf{S}$ exprimé dans la base $\left\{\underline{\mathbf{e}}_{i}\right\}=\underline{\mathbf{e}}_{i} \otimes \underline{\mathbf{e}}_{j} \otimes \ldots \otimes \underline{\mathbf{e}}_{m} \cdot\left\{\underline{\mathbf{e}}_{i}\right\}$ est la base tournée dont la rotation est utilisée pour le calcul des dérivées objectives. Le premier point principal du modèle est abordé au travers de cette équation. En effet, la très grande rigidité longitudinale des fibres requiert de suivre précisément leur direction sous peine d'accumuler d'importantes erreurs sur les contraintes. Pour cela, la base tournée, noté $\left\{\underline{\mathbf{f}}_{i}\right\}$, doit être attachée à la direction de fibre $\underline{\mathbf{f}}_{1}$, c'est-à-dire que l'on utilise une dérivée objective en rotation basée sur la rotation des fibres. Les deux autres vecteurs $\underline{\mathbf{f}}_{2}$ et $\underline{\mathbf{f}}_{3}$ de la base tournée doivent suivre les mouvements de corps rigide. $\underline{\mathbf{f}}_{2}$ est défini par projection dans le plan transverse du vecteur initial transporté par la déformation et $\underline{\mathbf{f}}_{3}$ par produit vectoriel $[9,10]$. Finalement, la matrice $[\mathbf{C}]_{f_{i}}$ utilisée dans l'équation ci-dessus est écrite dans cette base liée aux fibres, ce qui offre l'avantage de pouvoir distinguer ses composantes longitudinales et transversales afin de bien les identifier. Ce modèle a été comparé, pour des tests élémentaires, aux modèles classiques en grandes transformations (Jaumann, Green Naghdi), et il s'avère que pour un matériau à une direction de fibres, ces derniers ne garantissent pas un état de contrainte cohérent, contraitement au présent modèle. Un test de cisaillement sous tension est le plus discriminant.

Le comportement transverse est le second aspect important pour les analyses car il intervient sur les changements de forme des sections de mèches. On propose ici une approche dans laquelle les phénomènes sphériques et déviatoriques sont découplés dans le plan transverse aux fibres. Les observations de coupes encouragent cette approche : on distingue clairement deux modes de déformation de la mèche (voir Fig. 1b) : d'une part la compaction du réseau de fibres (visible en tension, par exemple) et d'autre part un réarrangement du réseau de fibres entraînant un changement de forme de la section transverse de la mèche. On considère par la suite que ces deux modes de déformation sont découplés, ce qui reste valide sous l'hypothèse d'isotropie dans ce plan [11].

La séparation en parties sphériques et déviatoriques de la partie transverse du tenseur des contraintes s'écrit :

$$
\begin{aligned}
{\left[\tilde{\boldsymbol{\sigma}}_{T}\right]_{f_{i}} } & =\left[\begin{array}{cr}
\sigma_{\mathrm{s}} & 0 \\
0 & \sigma_{\mathrm{s}}
\end{array}\right]+\left[\begin{array}{cr}
\sigma_{\mathrm{d}} & \sigma_{23} \\
\sigma_{23} & -\sigma_{\mathrm{d}}
\end{array}\right] \\
\text { avec } \quad \sigma_{\mathrm{s}} & =\frac{\sigma_{22}+\sigma_{33}}{2} \text { et } \sigma_{\mathrm{d}}=\frac{\sigma_{22}-\sigma_{33}}{2}
\end{aligned}
$$

Pour l'instant, le modèle est purement élastique (nonlinéaire) et le découplage proposé mène aux relations : $\sigma_{\mathrm{s}}=A \varepsilon_{\mathrm{s}} ; \sigma_{\mathrm{d}}=B \varepsilon_{\mathrm{d}} ; \sigma_{23}=C \varepsilon_{23}$ où $\varepsilon_{\mathrm{s}}$ et $\varepsilon_{\mathrm{d}}$ sont définis de la même manière que $\sigma_{\mathrm{s}}$ et $\sigma_{\mathrm{d}}$ et $A, B, C$ sont les coefficients élastiques. On peut montrer que $B=C$. Finalement le modèle de comportement transverse contient deux coefficients élastiques indépendants, ce qui correspond effectivement à un milieu isotrope bidimensionnel.

Pour compléter la description du modèle, la forme de $A$ et $B$ doit être spécifiée. Pour cela, nous nous basons sur des hypothèses physiques simples. Sous compaction, le matériau se rigidifie à la fois en comportement sphérique 
(a)
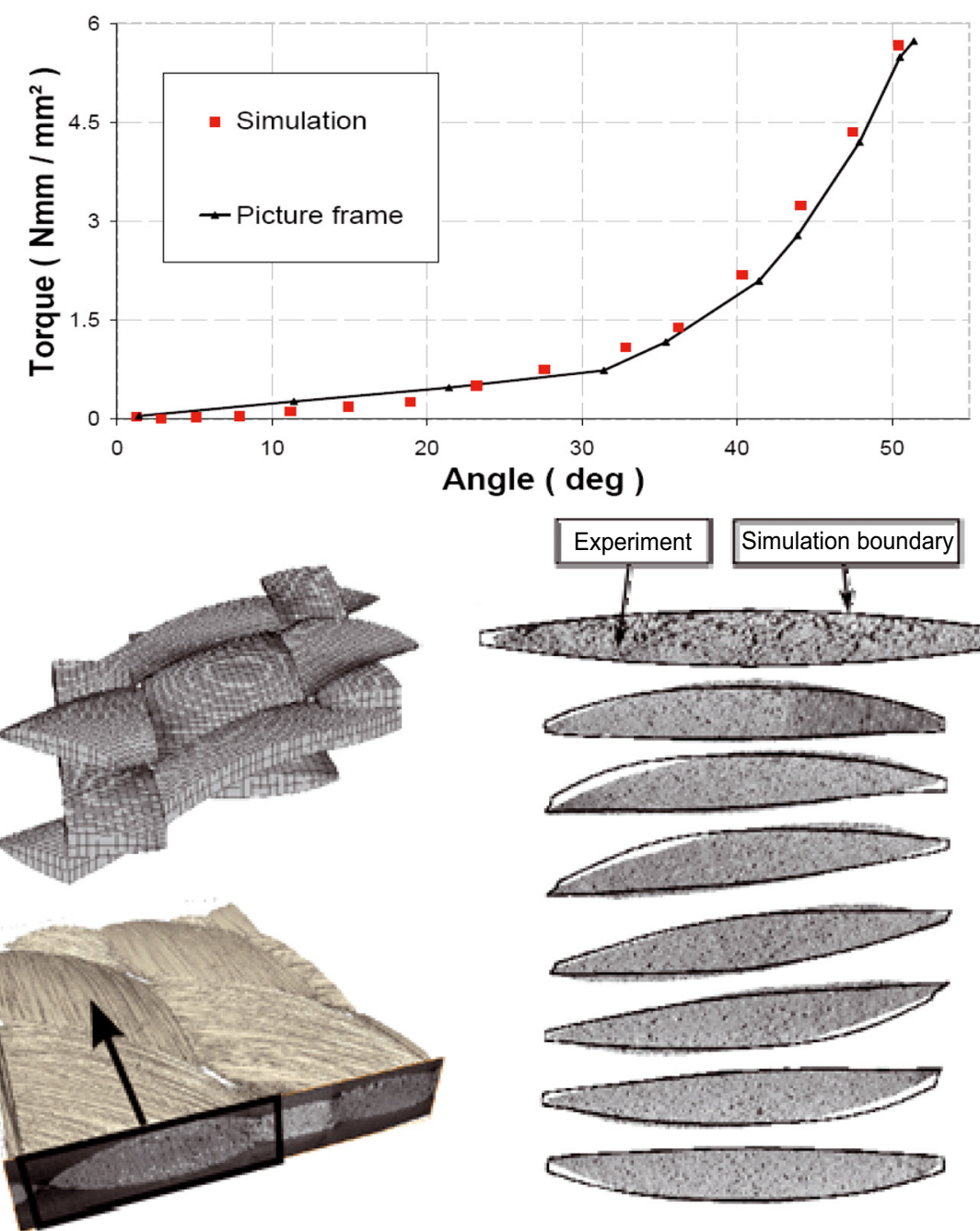

Fig. 1. Résultats de simulations de cisaillement. (a) Courbe de couple/angle de cisaillement. (b) Comparaison avec la tomographie.

et en comportement déviatorique en raison de la densification du paquet de fibres. Sous tension longitudinale également, le comportement sphérique doit être plus rigide. L'influence de la tension longitudinale sur le comportement déviatorique étant moins évidente, elle n'est pas considérée ici. À partir de ces hypothèses, la forme proposée pour les coefficients $A$ et $B$ est la suivante : $A=A_{0} \mathrm{e}^{-p \varepsilon_{\mathrm{s}}} \mathrm{e}^{n \varepsilon_{11}}$ et $B=B_{0} \mathrm{e}^{-p \varepsilon_{\mathrm{s}}}$.

\subsection{Simulations mésoscopiques}

En raison de la périodicité du renfort, et si le changement le permet, il convient de ne modéliser qu'une partie du renfort, appelée cellule élémentaire (voir Fig. 1b, cellule déformée) et d'appliquer des conditions aux limites adaptées. Dans certains cas, la géométrie minimale à modéliser peut être diminuée en raison de certaines symétries mais il ne s'agit pas du cas général, par exemple cela n'est pas possible en cisaillement.

Sur le plan mécanique, on s'intéresse aux résultats donnant le couple de cisaillement en fonction de l'angle de cisaillement. Ce genre de données peut être important lors de la conception d'un tissu, elles sont aussi utilisées pour alimenter les simulations de mise en forme de renforts de composites. En effet, ce comportement est à l'origine des plis, non souhaités, qui peuvent se former. La courbe présentée en figure 1a montre un accord satisfaisant avec des résultats expérimentaux mais il est à noter que les essais expérimentaux présentent une dispersion importante. La première partie de la courbe, de pente faible, correspond majoritairement à une rotation des mèches les unes relativement aux autres, alors que la seconde partie, de pente forte, traduit la mise en contact latéral des mèches qui mène à un «blocage » en cisaillement.

Sur le plan de la géométrie déformée qui peut être utilisée pour de nombreuses applications (dont en particulier l'évaluation de la perméabilité [12]), la reconstruction par tomographie a été faite à une résolution de $2,85 \mu \mathrm{m}$ pour un angle de cisaillement de $46^{\circ}$. La comparaison avec la simulation est donnée pour un jeu de sections le long d'une demi-période (Fig. 1b). On note que la section simulée est compactée assez fortement et que le modèle reproduit 

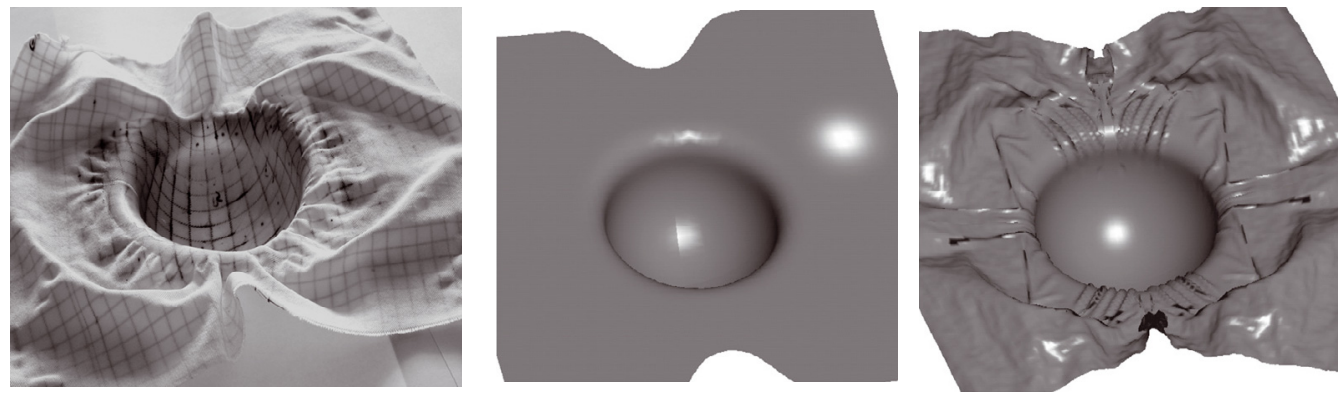

Fig. 2. Mise en forme d'un renfort déséquilibré. (G) Expérience, (C) simulation en tension, (D) simulation en tension et cisaillement.

les dissymétries et distorsions de la section transverse de mèche due au cisaillement du renfort.

\section{Simulation de la mise en forme des renforts fibreux}

La méthode des éléments-finis est aujourd'hui largement utilisée pour la simulation des procédés de formage des pièces métalliques et polymères. La difficulté consiste à traduire le comportement mécanique très particulier des renforts fibreux ici à l'échelle macroscopique. Compte tenu de la géométrie des tissus où l'épaisseur est petite devant les deux autres dimensions, les éléments utilisés se placent dans le cadre d'une hypothèse de coque ou de membrane. Dans ce dernier cas, la rigidité de flexion du tissu est négligée ce qui est en général une hypothèse raisonnable. Le comportement dans le plan est très particulier. Les fibres tissées lui confèrent une très forte anisotropie. Il est principalement défini par le comportement en traction biaxiale et en cisaillement. Deux approches sont possibles pour intégrer ce comportement spécifique dans une approche éléments-finis. La première consiste à définir un modèle continu en accord avec les particularités du tissu avec éventuellement de la résine [1,2]. Cette modélisation peut être introduite dans des éléments-finis de coques ou de membranes classiques. La définition d'un tel modèle est complexe et délicate compte tenu des spécificités apportées par le tissage en particulier lorsque les variations d'angles entre les mèches chaîne et trame sont grandes.

Une autre approche consiste à construire des élémentsfinis spécifiques aux tissés. Les éléments-finis utilisés dans l'exemple présenté ci-dessous sont dits semi-discrets $[5,6]$. Ils sont composés de mailles élémentaires tissées. Le comportement de chaque maille élémentaire est défini à partir de simulations à l'échelle mésoscopique telles que présentées en section 2, en tension biaxiale et en cisaillement plan. Les matrices de rigidité et efforts intérieurs nodaux sont obtenus par sommation sur chaque maille élémentaire. L'expression de chaque composante est simple car seules les rigidités principales sont prises en compte. Il en résulte des éléments-finis efficaces du point de vue de la rapidité de calcul.

Dans ce cadre, un exemple de simulation de mise en forme hémisphérique d'un renfort tissé est montré figure 2. Le tissu a la particularité d'être beaucoup plus rigide dans la direction chaîne que dans la direction trame. Le résultat expérimental de la mise en forme conduit à la déformée très dissymétrique suivant les directions chaîne et trame. Dans la partie utile hémisphérique, les carrés initiaux deviennent rectangulaires avec $L_{\text {trame }} / L_{\text {chaîne }}=1,8$ au centre du dôme. Les bords du tissé sont également très dissymétriques avec un fort avalement dans la direction chaîne contrairement à la direction trame. De nombreux plis sont présents dans la partie plane, aucun dans la partie utile hémisphérique. On montre le résultat obtenu par deux approches. Dans le premier cas (Fig. 2C), seule l'énergie de déformation de tension est prise en compte, alors que dans le deuxième cas (Fig. 2D), les comportements de tension et de cisaillement dans le plan sont pris en compte pour chaque maille élémentaire. Dans les deux cas la déformée de la partie utile hémisphérique est obtenue de façon cohérente avec le résultat expérimental $\left(L_{\text {trame }} / L_{\text {chaîne }}=1,87\right.$ et $1,83)$. On peut expliquer la faible influence du cisaillement par le fait que les variations d'angle dans cette zone restent inférieures à l'angle limite. L'énergie de cisaillement reste donc faible. La dissymétrie de la forme des bords de l'éprouvette est obtenue par les deux approches, mais de façon plus proche de l'expérience lorsque l'on tient compte du cisaillement. La différence la plus visible concerne l'obtention des plis. Ceux ci sont absents dans l'approche en tension uniquement alors qu'ils sont nombreux et correspondent correctement avec l'expérience lorsque le cisaillement est pris en compte. Ceci est lié au fait que dans certains zones l'angle limite de cisaillement est dépassé, d'où l'apparition d'instabilités compte tenu de l'importance de l'énergie de cisaillement. Les plis permettent d'obtenir des solutions où les angles entre chaînes et trames sont moins importants. Ces valeurs de distorsions sont en bon accord avec l'expérience alors que celles obtenues par la simulation en tension uniquement sont surévaluées. En conclusion la prise en compte du cisaillement permet de décrire les instabilités de plissement lorsque l'on a dépassé l'angle limite de cisaillement.

\section{Conclusions}

Les textiles sont des matériaux multi-échelles. Une simulation de la mise en forme à l'échelle macroscopique a 
été présentée. Elle utilise les comportements mécaniques obtenus en analysant la déformation d'une maille élémentaire dans des cas de tension biaxiale et de cisaillement dans le plan. La rigidité de cisaillement est de second ordre par rapport à celles de tension. Néanmoins elle est nécessaire pour obtenir l'apparition et le développement de plissements dans la simulation.

\section{Références}

[1] A.J.M. Spencer, Theory of fabric-reinforced viscous fluid, Compos. Part A 31 (2000) 1311-1321

[2] R.H.W. Ten Thije, R. Akkerman, J. Huetink, Large deformation simulation of anisotropic material using an updated Lagrangian finite element method, Comput. Meth. Appl. Mech. Eng. 196 (2007) 3141-3150

[3] B. Ben Boubaker, B. Haussy, J.F. Ganghoffer, Discrete models of woven structures. Macroscopic approach, Compos. Part B 38 (2007) 498-505

[4] D. Durville, Numerical simulation of entangled materials mechanical properties, J. Mat. Sci. 40 (2005) 5941-5948

[5] P. Boisse, B. Zouari, J.L. Daniel, Importance of in-plane shear rigidity in finite element analyses of woven fabric composite preforming, Compos. Part A 37-12 (2006) 2201-2212
[6] N. Hamila, P. Boisse, Simulations of textile composite reinforcement draping using a new semi-discrete three node finite element, Compos. Part B 39 (2008) 999-1010

[7] C. Truesdell, Hypo-elasticity, J. Rat. Mech. Anal. 4 (1955) 83-133

[8] T.J.R. Hughes, J. Winget, Finite rotation effects in numerical integration of rate constitutive equations arising in large deformation analysis, Int. J. Num. Meth. Eng. 15 (1980) 1862-1867

[9] P. Badel, E. Vidal-Salle, P. Boisse, Large deformation analysis of fibrous materials using rate constitutive equations, Comput. Structures 86 (2008) 1164-1175

[10] P. Badel, S. Gauthier, E. Vidal-Salle, P. Boisse, Rate constitutive equations for computational analyses of textile composite reinforcement mechanical behaviour during forming, Compos. Part A, On line, doi : 10.1016/j.compositesa.2008.04.015

[11] P. Badel, E. Vidal-Salle, E. Maire, P. Boisse, Simulation and tomography analysis of textile composite reinforcement deformation at the mesoscopic, Composites Science Technology 68 (2008) 2433-2440

[12] F. Loix, P. Badel, L. Orgéas, C. Geindreau, P. Boisse, Woven fabric permeability: from textile deformation to fluid flow mesoscale simulations, Composites Science Technology 68 (2008) 1624-1630 